\title{
Open charm and charmonium production at $\mathrm{LHCb}$
}

\author{
M. J. Charles ${ }^{\mathrm{a}}$, on behalf of the LHCb Collaboration. \\ ${ }^{a}$ Department of Physics, University of Oxford, \\ Denys Wilkinson Building, Keble Road, Oxford OX1 3RH, United Kingdom
}

We see copious production of charmed hadrons in the early LHCb data. The status and plans for charm production studies at $\mathrm{LHCb}$ are discussed, and preliminary signals are shown.

\section{INTRODUCTION}

The Large Hadron Collider (LHC) began colliding protons at a centre-of-mass energy of $7 \mathrm{TeV}$ at the end of March 2010. In the subsequent months, the luminosity has increased more or less exponentially: approximately $16 \mathrm{nb}^{-1}$ had been delivered to each experiment at the start of the BEACH2010 workshop, and at the time of writing these proceedings this has risen to approximately $2 \mathrm{pb}^{-1}$. Production of charmed hadrons is a natural topic to probe with these data samples: the cross sections are large enough to make precise measurements even with limited data samples, and early studies on charm distributions will improve our understanding of hadronic events at the LHC for subsequent analyses. It is anticipated that $\mathcal{O}\left(70 \mathrm{pb}^{-1}\right)$ will be delivered during the 2010 run, and $\mathcal{O}\left(1 \mathrm{fb}^{-1}\right)$ in 2011 [1]. In this note, we discuss the status of measurements of the cross section for prompt production of open and hidden charm mesons in $p p$ collisions at $\sqrt{s}=7 \mathrm{TeV}$ in bins of rapidity $y$ and transverse momentum $p_{t}$.

Charm and beauty hadrons are light compared to the collision energy at the LHC, so their production is concentrated in the forward region. This motivates the design of $\mathrm{LHCb}$ 2, a singlearm forward spectrometer, which instruments the angular range $15<\theta_{x}<300 \mathrm{mrad}$ and $15<$ $\theta_{y}<250 \mathrm{mrad}$, corresponding to approximately $2.0<\eta<5.2$, where $\theta_{x}$ and $\theta_{y}$ are the $x$ and $y$ projections of the polar angle, $\eta$ is the pseudorapidity, and the $z$-axis corresponds to the nominal beam direction. This coverage is complementary to the other large LHC experiments 3 445. The $\mathrm{LHCb}$ detector includes a silicon microstrip Vertex Locator (VELO) which is positioned at $5 \mathrm{~mm}$ from the beam during data-taking; five downstream tracking stations which use a combination of silicon pixels and drift chambers; two Ring Imaging Cherenkov (RICH) detectors with three different radiators to cover the momentum range $2<p<100 \mathrm{GeV} / c$; a calorimeter consting of a Scintillator Pad Detector (SPD) to detect charged particles, an electromagnetic calorimeter with a preshower detector, and a hadronic calorimeter; five muon stations; and a magnet with a field integral of $4 \mathrm{Tm}$.

Results at the BEACH2010 conference were presented with samples of $2-14 \mathrm{nb}^{-1}$. Where available, equivalent plots from larger data samples have been substituted in these proceedings.

\section{MEASUREMENT OF LUMINOSITY}

The integrated luminosity of the data is an essential input to cross section measurements. The approach used at $\mathrm{LHCb}$ is to calibrate quantities which are expected to be proportional to the instantaneous luminosity, such as the number of hits in the SPD, with runs in which the luminosity is measured directly. These calibrations can then be used to determine the integrated luminosity for a much larger data sample.

Two methods have been used to establish the instantaneous luminosity. First, the profiles of the beams can be measured directly in the VELO using beam-gas events, which are recorded continuously by the $\mathrm{LHCb}$ trigger at a low rate for 

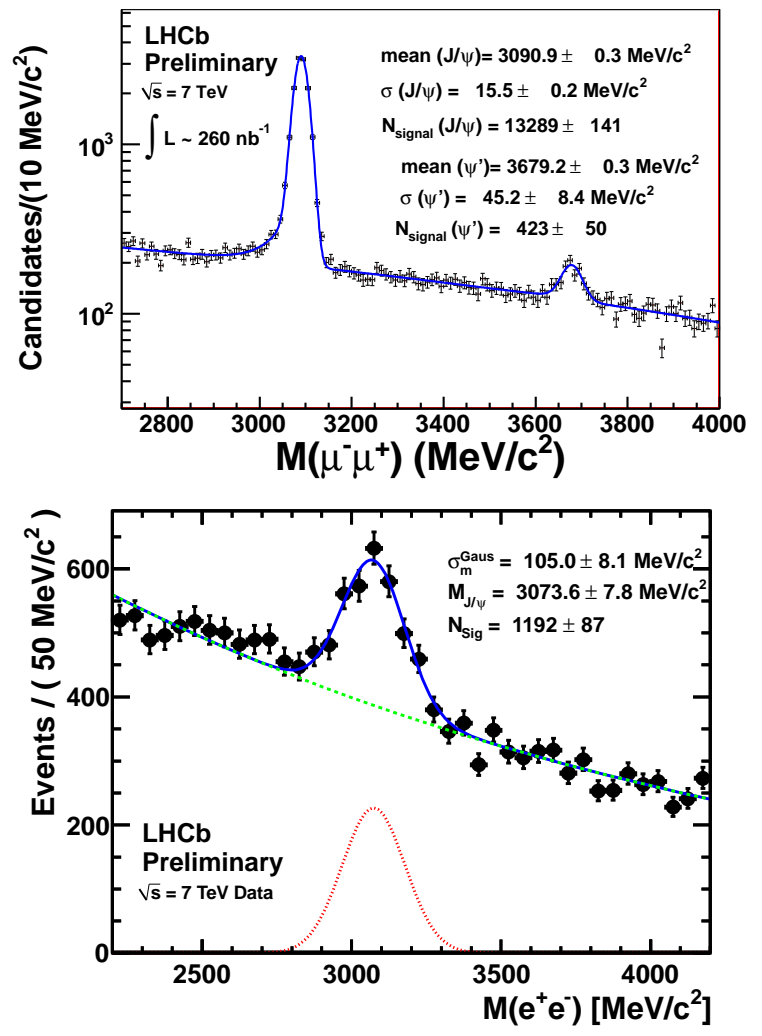

Figure 1. The invariant mass spectra for $\mu^{+} \mu^{-}$ (upper, $260 \mathrm{nb}^{-1}$ ) and $e^{+} e^{-}$(lower, $150 \mathrm{nb}^{-1}$ ). The $J / \psi$ and $\psi(2 S)$ resonances are visible. Note the logarithmic $y$-axis in the upper plot.

calibration purposes. Given the bunch-crossing frequency and beam currents, the luminosity can then be inferred [6]. Second, a dedicated run can be used for a Van der Meer scan [7. The uncertainty on the integrated luminosity when calibrated in this way at $\mathrm{LHCb}$ is estimated to be $10 \%$ at present.

\section{CHARMONIA}

The production mechanisms for $c \bar{c}$ bound states at hadron colliders are still far from understood; neither the Colour Singlet Model nor the Colour Octet Model fully describes the existing exper-

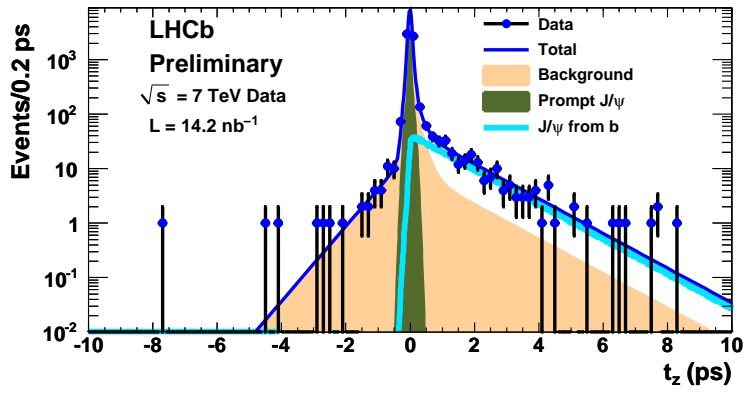

Figure 2. The pseudo-proper-time distribution for $J \psi \rightarrow \mu^{+} \mu^{-}$candidates in $14.2 \mathrm{nb}^{-1}$ of data.

imental data (see Ref. 8] and the references therein). The total cross section, the $p_{t}$ spectrum, and the polarization of $J^{P}=1^{-} c \bar{c}$ states are all of interest for probing this problem.

LHCb sees large signals for $J / \psi \rightarrow \mu^{+} \mu^{-}$and $e^{+} e^{-}$, and a smaller but significant signal for $\psi(2 S) \rightarrow \mu^{+} \mu^{-}$(Fig. 11). We can divide charmonium at the LHC into two classes: those produced at an event primary vertex (prompt), either directly or in the decay of a short-lived resonance such as a heavier $c \bar{c}$ state, and those produced in the decay of a long-lived $B$ hadron (secondary). $\mathrm{LHCb}$ plans to measure the cross section as a function of $y$ and $p_{t}$ for both classes of production separately. The two cases can be distinguished using the pseudo-proper-lifetime $t_{z}$ of the decay, defined as:

$t_{z} \equiv\left(d_{z} M\right) / p_{z}$,

where $d_{z}$ is the projection in the $z$ direction of the distance between the decay vertex and the associated event primary vertex, $M$ is the reconstructed invariant mass, and $p_{z}$ is the $z$ component of the momentum. For prompt production, $t_{z}$ is described by a narrow peak at zero with width equal to the resolution; for secondary production, it is described by an exponential with effective lifetime approximately equal to the average $b$-hadron proper lifetime. This is illustrated for $J / \psi$ in Fig.2 The fit also includes a component for combinatoric background, determined from a mass sideband above the $J / \psi$. The data clearly 

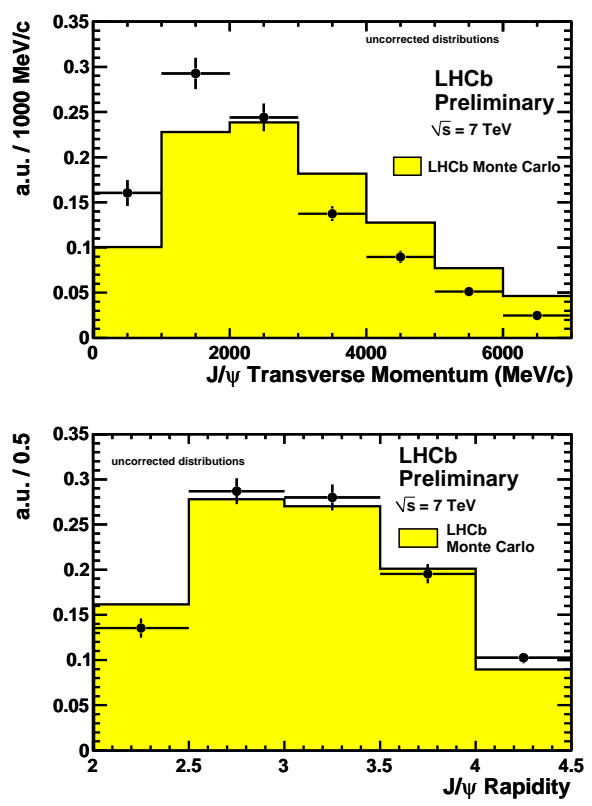

Figure 3. $J / \psi$ yields from $14 \mathrm{nb}^{-1}$ of data, not corrected for efficiency and including both prompt and secondary components. The upper plot shows the yield in bins of $p_{t}$ for $2.5<y<4.0$, and the lower plot shows the yield in bins of $y$ for $0<p_{t}<12 \mathrm{GeV} / c$. The points with error bars show the measured yield with statistical uncertainties only, and for comparison the shaded histogram shows the distribution seen in the $\mathrm{LHCb}$ Monte Carlo.

require a secondary component to be present.

A measurement in fine-grained bins $(0.5$ in $y$ $\times 1 \mathrm{GeV} / c$ in $\left.p_{t}\right)$ will require $\mathcal{O}\left(10-20 \mathrm{pb}^{-1}\right)$, but preliminary studies in one-dimensional bins are underway: the uncorrected yields seen in onedimensional bins are shown in Fig. 3. Firm conclusions cannot be drawn without systematic uncertainties, but these initial results illustrate the potential improvements from retuning the Monte Carlo generator with early data.
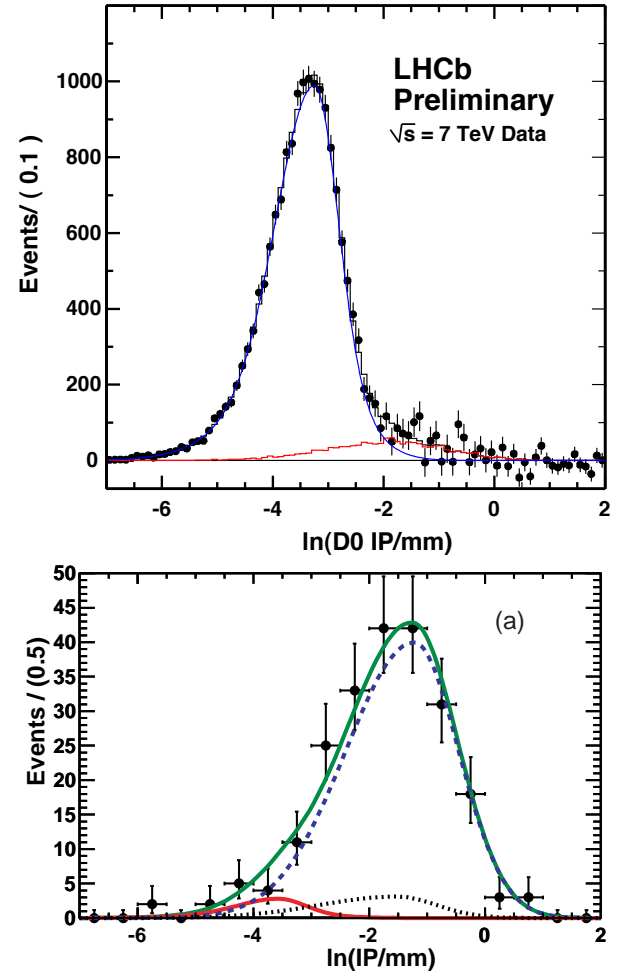

Figure 4. The logarithm of the $D^{0}$ impact parameter distribution for $B \rightarrow D^{0}\left(K^{-} \pi^{+}\right) \mu^{ \pm}$candidates. The upper plot $\left(3 \mathrm{nb}^{-1}\right)$ shows the background-subtracted distribution for inclusive $D^{0}$ candidates; the histograms indicate the fitted prompt and secondary components. The lower plot $\left(11 \mathrm{nb}^{-1}\right)$ shows $D^{0}$ candidates after requiring a $B \rightarrow D^{0} \mu^{+}$tag, which suppresses the prompt component strongly; the curves indicate the components of the fit: prompt (solid), secondary (dashed), background (dotted), and their sum (solid).

\section{CHARMED MESONS}

Similar production studies are in progress for the charmed mesons $D^{0}, D^{+}, D^{*+}$, and $D_{s}^{+}$. The excellent signal quality is illustrated in Fig. 5. As with the $J / \psi$, both prompt and secondary production are expected at LHCb. However, because 

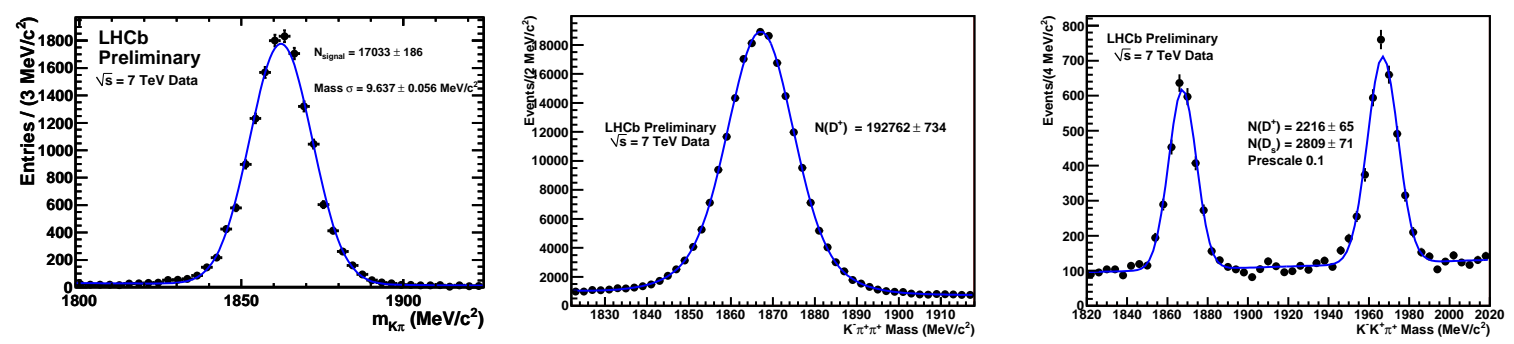

Figure 5. Invariant mass spectra for charmed mesons. From left to right: $m\left(K^{-} \pi^{+}\right)$for $D^{*+} \rightarrow$ $D^{0}\left(K^{-} \pi^{+}\right) \pi^{+} ; m\left(K^{-} \pi^{+} \pi^{+}\right) ; m\left(K^{-} K^{+} \pi^{+}\right)$. The first two plots use $125 \mathrm{nb}^{-1}$, and the third uses $13 \mathrm{nb}^{-1}$.

the $D^{0}, D^{+}$, and $D_{s}^{+}$fly a significant distance at $\mathrm{LHCb}$, separating the two sources is more complicated. The discriminating variable used is the impact parameter (IP) of the $D$ candidate with respect to the associated event primary vertexprompt charm points back to the primary vertex and so its IP distribution is dominated by the resolution, whereas secondary charm in general has a much larger IP. This is illustrated in Fig 4 . By fitting both the invariant mass spectrum and the $\log (\mathrm{IP})$ distribution, the production rate of prompt charm mesons can be measured. Inclusive cross section measurements in bins of $p_{y}$ and $y$ using this technique are now underway.

\section{OUTLOOK}

The production measurements described above are in progress and will be finalized over the coming months. In the meantime, the LHC continues to deliver ever-increasing luminosities. With the much larger data samples expected by the end of the 2010 run, $\mathrm{LHCb}$ will be able to search for new physics in charm decays. Three key analyses will be measurements of $y_{C P}$ and $A_{\Gamma}$ with $D^{0} \rightarrow K^{-} K^{+}$and $K^{-} \pi^{+}$; searches for direct CP violation in $D^{+} \rightarrow K^{-} K^{+} \pi^{+}$and $\pi^{-} \pi^{+} \pi^{+}$; and a search for $D^{0} \rightarrow \mu^{+} \mu^{-}$. These studies will benefit greatly from the combination of copious charm production, a large boost (especially valuable for $y_{C P}$ and $A_{\Gamma}$ ), and excellent charged particle iden- tification across the full momentum range.

\section{REFERENCES}

1. S. Myers, XXXV International Conference on High Energy Physics, Paris, France, July 2228, 2010.

2. LHCb Collaboration, A. A. Alves et al., J. Instrum. 3 (2008) S08005.

3. ALICE Collaboration, K. Aamodt et al., J. Instrum. 3 (2008) S08002.

4. ATLAS Collaboration, G. Aad et al., J. Instrum. 3 (2008) S08003.

5. CMS Collaboration, S Chatrchyan et al., J. Instrum. 3 (2008) S08004.

6. H. Burkhardt and P. Grafstrom, CERN-LHCPROJECT-REPORT-1019.

7. S. van der Meer, CERN-ISR-PO-68-31.

8. J. P. Lansberg, Int. J. Mod. Phys. A 21 (2006) 3857 . 